\title{
Complementation of a temperature sensitive Escherichia coli rpoD mutation using Lactobacillus sigma factors
}

\author{
James D. Winkler*and Katy C. Kao ${ }^{\dagger}$
}

March 1, 2014

\begin{abstract}
Housekeeping sigma factors in the $\sigma^{70}$ family, as components of the RNA polymerase holoenzyme, are responsible for regulating transcription of genes related to vegetative growth. While these factors are well understood in model organisms such as Escherichia coli and Bacillus subtilis, little experimental work has focused on the sigma factors in members of the Lactobacillus genus such as Lactobacillus brevis and Lactobacillus plantarum. This study evaluates the ability of putative $\sigma^{70}$ proteins from L. brevis $\left(\sigma_{L b}^{70}\right)$ and L. plantarum $\left(\sigma_{L p}^{70}\right)$ to complement a temperature sensitive mutation in the E. coli $285 \mathrm{c} \sigma^{70}$ protein. This report is the first to show that these heterologous sigma factors were capable of restoring the viability of $E$. coli 285 c for growth at $40-43.5^{\circ} \mathrm{C}$, indicating the $\sigma_{L b}^{70}$ and $\sigma_{L p}^{70}$ are capable of initiating transcription in a complex with the E. coli $285 \mathrm{c}$ RNA polymerase. These heterologous sigma factors may therefore be useful for improving biochemical knowledge of the sigma factor family or for use in the expression of hetereologous genomic libraries.
\end{abstract}

\footnotetext{
${ }^{*}$ Department of Chemical Engineering, Texas A\&M University, College Station, Texas, USA

${ }^{\dagger}$ Department of Chemical Engineering, Texas A\&M University, College Station, Texas, USA. Address correspondence to Katy C. Kao, kao.katy@mail.che.tamu.edu
} 


\section{Introduction}

Two members of the Lactobacillus genus, Lactobacillus brevis and Lactobacillus plantarum, have been shown to possess many desirable characteristics including superior acid, butanol, and ethanol tolerance that are of interest for industrial usage [1-5]. These species have only recently been analyzed [6-9] and as such their biochemical and genetic corpus is lacking compared to the model organism Escherichia coli. In particular, the transcriptional programs of the Lactobacilli and regulation therein under many conditions are poorly understood, though characterization efforts are underway [10-12]. Housekeeping sigma factors $\left(\sigma^{70}\right.$ or $\left.\sigma^{A}\right)$ [13] such as those putatively found in L. brevis and L. plantarum genomes, given their role in controlling gene transcription through promoter recognition during exponential growth, play an important role in the physiology of these organisms. Experimental study of the Lactobacilli sigma factors will improve our understanding of how these factors control the Lactobacillus transcriptional program.

The importance of sigma factors arises from their transient contact with the eubacterial RNA polymerase core enzyme $\left(\alpha_{2} \beta \beta^{\prime} \omega\right)$ that allows RNAP to specifically recognize promoter sequences upstream of genes [14-18]. Without these proteins, gene transcription is impossible. Sigma factors are divided into protein families based on structural homology, with factors responsible for regulating gene expression during exponential growth belonging to group 1 [13, 15, 19, 20]; by acting in concert, the various sigma factors within an organism effectively divide the transcriptional program of an organism into multiple sub-routines capable of responding to environmental conditions rapidly [21]. While the understanding of the biological roles of sigma factors found in well characterized organisms is extensive [22-26], the lack of experimental data on L. brevis and L. plantarum physiology means that the putative sigma factors in these organisms are understood only by analogy to the related Bacillus subtilis [27] and through sequence analysis of known promoters. In light of their role in regulating genes critical for growth, the housekeeping sigma factors of L. brevis $\left(\sigma_{L B}^{70}\right)$ and L. plantarum $\left(\sigma_{L P}^{70}\right)$ are the best candidates for in vivo examination to better understand the transcriptional programs of these organisms.

Analysis of the Lactobacilli sigma factors poses significant challenges given the relative paucity of genetic tools available for these organisms. We chose to examine the behavior of $\sigma_{L b}^{70}$ and $\sigma_{L p}^{70}$ within E. coli as a result, in spite of the significant differences between the organisms. This 
approach has been successful in the past with non- E. coli housekeeping sigma factors [28-32] and other factors regulating flagellar production $\left(\sigma^{F}\right)$ and response to extracellular stress $\left(\sigma^{E}\right)[33-35]$ in complementing E. coli null phenotypes. Sigma factors that are too structurally divergent from those found in E. coli tend to be non-functional in vivo [36]. Given the diversity of heterologous sigma factors that are functional in E. coli, there must exist a significant amount of functional flexibility of $\sigma$-RNA polymerase interactions. An improved understanding of the minimal sigma factor sequence required for functioning would improve our understanding of both a basic aspect of bacterial physiology and enhance efforts for sigma factor engineering.

To that end, this work analyzes the functionality of $\sigma_{L B}^{70}$ and $\sigma_{L P}^{70}$ within an E. coli with a temperature sensitive defect in the native $\sigma_{E c, t s}^{70}$ protein [37-40]. We first compare the structure of Lactobacilli sigma factors to other functional, heterologous sigma factors that have been expressed in E. coli in the past to detect any common features amongst these proteins. Two expression plasmids containing the Lactobacilli sigma factors promoter are then constructed and are shown to complement the defective $\sigma_{E c, t s}^{70}$ under non-permissive conditions. Potential applications for expression of foreign promoters in metabolic engineering are subsequently discussed.

\section{Experimental Procedures}

\section{Bacterial strains and growth conditions}

E. coli (BW25113 and 285c), Lactobacillus brevis (ATCC 367), and Lactobacillus plantarum (WCFS1) were used in this study. E. coli cultures were grown in LB broth at $37^{\circ} \mathrm{C} ; 50 \mu \mathrm{g} / \mathrm{mL}$ of streptomycin was added as necessary. The E. coli mutant strain $285 \mathrm{c}$ with a temperature sensitive defect in its $\sigma^{70}$ protein and the parent strain P9OA5c [37, 41, 42] were generously provided by Robert Calendar (UC Berkeley) and cultured at $30^{\circ} \mathrm{C}$ in $\mathrm{LB}$ media and $25 \mu \mathrm{g} / \mathrm{ml}$ streptomycin (if needed) to prevent selection of revertant mutations in $\sigma^{H}[40]$. For the complementation assay, the E. coli 285c strain were transformed with empty pCLE vector backbone or plasmids containing L. brevis or L. plantarum $\sigma$-factors. Cultures were grown overnight at $30^{\circ} \mathrm{C}$ in $\mathrm{LB}$ media with $25 \mu \mathrm{g} / \mathrm{ml}$ streptomycin. The growth of the P9OA5c, 285c/pCLE, 285c/pCLE-Lp, and $285 \mathrm{c} / \mathrm{pCLE}-\mathrm{Lb}$ strains was monitored spectrophotomerically at $42{ }^{\circ} \mathrm{C}$ using a TECAN plate 
reader to assess the complementation effect of the heterologous sigma factors. Batch studies of growth at $43.5-46^{\circ} \mathrm{C}$ were conducted in a shaking water bath at $200 \mathrm{rpm}$.

\section{$\sigma$-factor selection}

Putative $\sigma^{70}$ growth factors were identified on the basis of sequence annotation (L. plantarum: LP-1926, L. brevis: LVIS-0756) [43] for study. Heterologous sigma factors were placed under the control of $P_{l a c}$ with the native $\sigma_{E c}^{70}$ ribosome binding site to ensure equivalent translation efficiency. Primers for these sequences were designed from the E. coli-MG1655, L. brevis ATCC 367, and L. plantarum WCFS1 strains using Primer-BLAST [43] (Table S1). Sequence alignments of E. coli, Lactobacilli, and other sigma factor proteins [28-31, 44] using Clustal Omega $[45]$.

\section{Genetic manipulation}

The low copy number pCL1920 plasmid [46] was used to express the $\sigma^{70}$-factors from L. plantarum and L. brevis in E. coli. First, the 300 base pair region upstream of the native E. coli $\sigma^{70}$ gene containing the native RBS was introduced into pCL1920 to create pCL1920-EcS70 (pCLE). Note that the pCLE plasmid does not contain the $\sigma_{E c}^{70}$ gene. Subsequently, each of the $\sigma$ factor gene was amplified directly from the corresponding genomic DNA and subsequently purified using the Clean and Concentrator kit (Zymo Research). The fragments were digested with the appropriate restriction enzymes and ligated into the digested pCLE plasmid using T4 DNA ligase (New England Biolabs) to create pCLE- $\sigma_{L b}^{70}$ (pCLE-Lb) and pCLE- $\sigma_{L p}^{70}$ (pCLE-Lp). These expression plasmids were then introduced into E. coli (BW25113 and 285c) by electroporation (BioRad GenePulser XL) and plated onto selective media. All cloning PCR steps were performed using the Phusion polymerase (Finnzymes) and the constructs verified by sequencing (MCLAB Inc). 


\section{Results}

\section{Sequence Conservation in Heterologous Sigma Factors}

In order to better understand why the Lactobacilli sigma factors are functional, we compared the sequences of all housekeeping sigma factors that have been expressed in E. coli to this date to identify any conserved structural features (Figure 3). All aligned sigma factors shared at least $44 \%$ amino acid similarity, and the L. plantarum and L. brevis factors are $66 \%-70 \%$ similar. Based on sequence alignments, domain 1 and the linker connecting it to domain 2 [13] appear to be entirely absent from the gram positive sigma factors, though there is a high degree of conservation in domains 2 and 4 in all of the heterologously expressed proteins. These sigma factor domains control interactions with RNA polymerase and DNA, and are therefore critical for promoter recognition [13]. Housekeeping sigma factors from Caulobacter crescentus, Rickettsia prowazekii, Microcystis aeruginosa, and Bordetella pertussi were also very similar to E. coli $\sigma^{70}$ in domains 2 and 4 , and all but M. aeruginosa contained a poorly conserved domain 1 . Based on the data today, it appears that the only requirement for functional housekeeping sigma factor in E. coli may be sufficient similarity between domains 2 and 4 in the native and heterologous proteins [19]

\section{Lactobacilli sigma factors complement native $\sigma^{70}$ defects}

The first step in evaluating the properties of the heterologous sigma factors is to assess whether the sigma factors from L. brevis and L. plantarum are capable of complementing a temperature sensitive defect in the $\sigma_{E c, t s}^{70}$ protein found in the E. coli 285c strain [37], as has been done repeatedly in the past for other heterologous sigma factors [29-31, 44, 47, 48]. First, low-copy plasmids containing the Lactobacillus sigma factors were introduced into E. coli 285c (see Methods and Materials). The growth kinetics of the 285c/pCLE-Lb, 285c/pCLE-Lp, 285c/pCLE, and the parent strains (P9OA5c) as shown in Figure 1 are quite similar at the permissive temperature (30 ${ }^{\circ} \mathrm{C}$ ), indicating that there is no defect in $\sigma_{E c, t s}^{70}$ activity at low temperatures. However, subjecting the strains to the non-permissive temperature $\left(42^{\circ} \mathrm{C}\right)$ reveals significant differences between the strains (Figure 2). Little to no growth is seen in the 285c/pCLE strain, while the 285c/pCLE-Lb 
and $285 \mathrm{c} / \mathrm{pCLE}-\mathrm{Lp}$ strains containing the heterologous sigma factors are not only viable, but indeed grow fairly robustly. The doubling time of the 285c/pCLE-Lb and 285c/pCLE-Lp strains are significantly greater than that of the parent strain ( 255 min versus 108 min for P9OA5c), suggesting that while $\sigma_{L B}^{70}$ and $\sigma_{L P}^{70}$ can restore viability at elevated temperatures, the growth rate of $285 \mathrm{c}$ is still impaired overall due to imperfect complementation or differences in promoter specificity compared to the native $\sigma^{70}$. These results strongly support the conclusion that the L. brevis and L. plantarum sigma factors can successfully bind to the $E$. coli RNA polymerase and initiate transcription in vivo effectively, as otherwise the elevated temperature would have permanently arrested growth in all strains.

There are several possible physiological roles for the heterologous sigma factors within E. coli that can be inferred from this complementation effect. Firstly, these proteins may in fact act as direct replacements for $\sigma_{E c}^{70}$ factor, enabling the transcription of genes required for exponential growth at high temperatures. This hypothesis is lent support by the fact that the $\sigma^{70}$ promoter consensus sequences for lactic acid bacteria including those of L. brevis and L. plantarum are close to that of E. coli [49-54]. Efficient transcription of several E. coli promoters by the Lactobacillus acidophilus RNA polymerase holoenzyme has also been previously identified [55], providing additional weight to this interpretation. Suppressor mutations, such as those in rpoH or proteases, increase the stability of $\sigma_{E c, t s}^{70}$ in vivo and restore the viability of $285 \mathrm{c}$ at elevated temperatures [56]. To test for compensatory mutations that restore growth at elevated temperatures, the ability of the $285 \mathrm{c} / \mathrm{pCLE}-\mathrm{Lb}$ and $285 \mathrm{c} / \mathrm{pCLE}-\mathrm{Lp}$ to grow at $43.5^{\circ} \mathrm{C}$ was assayed in batch cultures. Both strains grow robustly at $43.5{ }^{\circ} \mathrm{C}$ (data not shown), indicating that they most likely do not have defects in proteolysis related genes that would stabilize $\sigma_{E c, t s}^{70}$. It is still possible that rpoH suppressor mutations exist in the strains but this phenotypic reversion is extremely rare (frequency $\approx 10^{-8}$ ) and unlikely to have occurred in independent replicates of $285 \mathrm{c} / \mathrm{pCLE}-\mathrm{Lb}$ and $285 \mathrm{c} / \mathrm{pCLE}-\mathrm{Lp}$ simultaneously. It was also recently demonstrated that expression of $\sigma_{L p}^{70}$ confers enhanced transcription of L. planatarum gene fragments [32], providing further evidence that these sigma factors are functional when heterologously expressed. Based on these growth kinetics and small likelihood of undetected compensatory mutations, we conclude that the Lactobacillus $\sigma^{70}$ proteins are responsible for the observed complementation effect. 


\section{Conclusions}

We have demonstrated that two primary sigma factors from L. brevis and L. plantarum are able to complement a temperature sensitive growth defect in E. coli $285 \mathrm{c}$ that results from loss of the native $\sigma^{70}$ activity at elevated temperatures. This finding appears to be one of the first demonstrating complementation with a Gram-positive primary sigma factor, and therefore provides additional insight into the plasticity of both the RNA polymerase core enzyme and the sigma factors themselves. Techniques that modify the native E. coli transcriptional program such as global transcription machinery engineering (gTME) may also see a benefit from the use of the Lactobacilli sigma factors given their ability to mediate transcription in complex with RNA polymerase. Engineering E. coli to transcribe L. brevis or L. plantarum genes effectively may also depend on the expression of the sigma factors in situations where compatible promoters cannot be introduced, such as during the protoplast fusion of these organisms.

\section{Acknowledgements}

We gratefully acknowledge funding from the National Science Foundation Graduate Student Fellowship program, US NSF grant CBET-1032487, and the Texas Experimental Engineering Station, as well as experimental assistance from Maria Priscilla Almario Falls. 


\section{References}

[1] R.S. Gold, MM Meagher, R. Hutkins, and T. Conway. Ethanol tolerance and carbohydrate metabolism in Lactobacilli. Journal of Industrial Microbiology and Biotechnology, 10(1):4554, 1992.

[2] D. Fiocco, V. Capozzi, P. Goffin, P. Hols, and G. Spano. Improved adaptation to heat, cold, and solvent tolerance in Lactobacillus plantarum. Applied Microbiology and Biotechnology, 77(4):909-915, 2007.

[3] E.P. Knoshaug and M. Zhang. Butanol tolerance in a selection of microorganisms. Applied Biochemistry and Biotechnology, 153(1):13-20, 2009.

[4] J.H. Kim, S.P. Shoemaker, and D.A. Mills. Relaxed control of sugar utilization in Lactobacillus brevis. Microbiology, 155(4):1351, 2009.

[5] W. Guo, W. Jia, Y. Li, and S. Chen. Performances of Lactobacillus brevis for Producing Lactic Acid from Hydrolysate of Lignocellulosics. Applied Biochemistry and Biotechnology, 161(1):124-136, 2010.

[6] M. Kleerebezem, J. Boekhorst, R. Van Kranenburg, D. Molenaar, O.P. Kuipers, R. Leer, R. Tarchini, S.A. Peters, H.M. Sandbrink, M.W.E.J. Fiers, et al. Complete genome sequence of Lactobacillus plantarum WCFS1. Proceedings of the National Academy of Sciences, 100(4):1990, 2003.

[7] B. Teusink and E.J. Smid. Modelling strategies for the industrial exploitation of lactic acid bacteria. Nature Reviews Microbiology, 4(1):46-56, 2006.

[8] K. Makarova, A. Slesarev, Y. Wolf, A. Sorokin, B. Mirkin, E. Koonin, A. Pavlov, N. Pavlova, V. Karamychev, N. Polouchine, et al. Comparative genomics of the lactic acid bacteria. Proceedings of the National Academy of Sciences, 103(42):15611, 2006.

[9] K.S. Makarova and E.V. Koonin. Evolutionary genomics of lactic acid bacteria. Journal of Bacteriology, 189(4):1199, 2007. 
[10] D. Molenaar, F. Bringel, F.H. Schuren, W.M. De Vos, R.J. Siezen, and M. Kleerebezem. Exploring Lactobacillus plantarum genome diversity by using microarrays. Journal of Bacteriology, 187(17):6119, 2005.

[11] B. Pieterse, R.J. Leer, F.H.J. Schuren, and M.J. van der Werf. Unravelling the multiple effects of lactic acid stress on Lactobacillus plantarum by transcription profiling. Microbiology, $151(12): 3881,2005$.

[12] B. Teusink, A. Wiersma, D. Molenaar, C. Francke, W.M. de Vos, R.J. Siezen, and E.J. Smid. Analysis of growth of Lactobacillus plantarum WCFS1 on a complex medium using a genome-scale metabolic model. Journal of Biological Chemistry, 281(52):40041, 2006.

[13] MS Paget and J.D. Helmann. The $\sigma^{70}$ family of sigma factors. Genome Biology, 4(203):20034, 2003.

[14] CA Gross, C. Chan, A. Dombroski, T. Gruber, M. Sharp, J. Tupy, and B. Young. The functional and regulatory roles of sigma factors in transcription. In Cold Spring Harbor Symposia on Quantitative Biology, volume 63, page 141. Cold Spring Harbor Laboratory Press, 1998.

[15] M. Wosten. Eubacterial sigma-factors. FEMS Microbiology Reviews, 22(3):127-150, 1998.

[16] T.M. Gruber and C.A. Gross. Multiple Sigma Subunits and the Partitioning of Bacterial Transcription Space. Annual Reviews in Microbiology, 57(1):441-466, 2003.

[17] D.F. Browning and S.J.W. Busby. The regulation of bacterial transcription initiation. Nature Reviews Microbiology, 2(1):57-65, 2004.

[18] S.J.W. Busby. More pieces in the promoter jigsaw: recognition of-10 regions by alternative sigma factors. Molecular Microbiology, 72(4):809-811, 2009.

[19] J.D. Helmann and M.J. Chamberlin. Structure and function of bacterial sigma factors. Annual Review of Biochemistry, 57(1):839-872, 1988.

[20] M. Lonetto, M. Gribskov, and CA Gross. The $\sigma^{70}$ family: sequence conservation and evolutionary relationships. Journal of Bacteriology, 174(12):3843, 1992. 
[21] J.D. Heimann. The extracytoplasmic function (ECF) sigma factors. Advances in Microbial Physiology, 46:47-110, 2002.

[22] J.T. Wade, D.C. Roa, D.C. Grainger, D. Hurd, S.J.W. Busby, K. Struhl, and E. Nudler. Extensive functional overlap between $\sigma$ factors in Escherichia coli. Nature Structural \& Molecular Biology, 13(9):806-814, 2006.

[23] G. Nonaka, M. Blankschien, C. Herman, C.A. Gross, and V.A. Rhodius. Regulon and promoter analysis of the E.coli heat-shock factor, $\sigma 32$, reveals a multifaceted cellular response to heat stress. Genes \& Development, 20(13):1776, 2006.

[24] T. Dong, M.G. Kirchhof, and H.E. Schellhorn. RpoS regulation of gene expression during exponential growth of Escherichia coli K12. Molecular Genetics and Genomics, 279(3):267$277,2008$.

[25] S.P. Haugen, W. Ross, and R.L. Gourse. Advances in bacterial promoter recognition and its control by factors that do not bind DNA. Nature Reviews Microbiology, 6(7):507-519, 2008 .

[26] S.M. Chiang and H.E. Schellhorn. Evolution of the RpoS Regulon: Origin of RpoS and the Conservation of RpoS-Dependent Regulation in Bacteria. Journal of Molecular Evolution, pages $1-15,2010$.

[27] W.G. Haldenwang. The sigma factors of Bacillus subtilis. Microbiology and Molecular Biology Reviews, 59(1):1, 1995.

[28] L.P. Aniskovitch and H.H. Winkler. Rickettsia prowazekii sigma factor $\sigma^{73}$ can be overexpressed in Escherichia coli and promotes RNA polymerase binding and transcription. Microbiology, 142(4):901, 1996.

[29] P. Steffen, S. Goyard, and A. Ullmann. The Bordetella pertussis sigma subunit of RNA polymerase confers enhanced expression of fha in Escherichia coli. Molecular Microbiology, 23(5):945-954, 1997. 
[30] D. Beier, G. Spohn, R. Rappuoli, and V. Scarlato. Functional analysis of the Helicobacter pylori principal sigma subunit of RNA polymerase reveals that the spacer region is important for efficient transcription. Molecular Microbiology, 30(1):121-134, 1998.

[31] J. Malakooti and B. Ely. Principal sigma subunit of the Caulobacter crescentus RNA polymerase. Journal of Bacteriology, 177(23):6854, 1995.

[32] Kyle A Zingaro, Sergios Nicolaou, Yongbo Yuan, and Eleftherios Terry Papoutsakis. Exploring the heterologous genomic space for building, stepwise, complex, multicomponent tolerance to toxic chemicals. ACS Synthetic Biology, 2014.

[33] Y.F. Chen and J.D. Helmann. Restoration of motility to an Escherichia coli fliA flagellar mutant by a Bacillus subtilis sigma factor. Proceedings of the National Academy of Sciences, 89(11):5123, 1992 .

[34] H. Yu, MJ Schurr, and V. Deretic. Functional equivalence of Escherichia coli $\sigma^{E}$ and Pseudomonas aeruginosa AlgU: E.coli rpoE restores mucoidy and reduces sensitivity to reactive oxygen intermediates in alg $U$ mutants of $P$. aeruginosa. Journal of Bacteriology, $177(11): 3259,1995$.

[35] K. Heuner, J. Hacker, and B.C. Brand. The alternative sigma factor $\sigma^{28}$ of Legionella pneumophila restores flagellation and motility to an Escherichia coli fliA mutant. Journal of Bacteriology, 179(1):17, 1997.

[36] D. Vingadassalom, A. Kolb, C. Mayer, T. Rybkine, E. Collatz, and I. Podglajen. An unusual primary sigma factor in the Bacteroidetes phylum. Molecular Microbiology, 56(4):888-902, 2005.

[37] J.D. Harris, J.S. Heilig, I.I. Martinez, R. Calendar, and L.A. Isaksson. Temperature-sensitive Escherichia coli mutant producing a temperature-sensitive sigma subunit of DNA-dependent RNA polymerase. Proceedings of the National Academy of Sciences, 75(12):6177, 1978.

[38] Y. Nakamura, T. Osawa, and T. Yura. Intragenic localization of amber and temperaturesensitive rpoD mutations affecting RNA polymerase sigma factor of Escherichia coli. Molecular and General Genetics, 189(2):193-198, 1983. 
[39] R.M. Blumenthal and PP Dennis. Regulation of ribonucleic acid polymerase synthesis during restriction of an Escherichia coli mutant temperature sensitive for transcription factor sigma. Journal of Bacteriology, 142(3):1049, 1980.

[40] A.D. Grossman, YN Zhou, C. Gross, J. Heilig, G.E. Christie, and R. Calendar. Mutations in the rpoH (htpR) gene of Escherichia coli K-12 phenotypically suppress a temperaturesensitive mutant defective in the sigma 70 subunit of RNA polymerase. Journal of Bacteriology, 161(3):939, 1985.

[41] L.A. Isaksson, S.E. Skold, J. Skjoldebrand, and R. Takata. A procedure for isolation of spontaneous mutants with temperature sensitive synthesis of RNA and/or protein. Molecular and General Genetics, 156(3):233-237, 1977.

[42] H. Liebke, C. Gross, W. Walter, and R. Burgess. A new mutation rpoD800, affecting the sigma subunit of E.coli RNA polymerase is allelic to two other sigma mutants. Molecular and General Genetics, 177(2):277-282, 1980.

[43] S.F. Altschul, W. Gish, W. Miller, E.W. Myers, and D.J. Lipman. Basic local alignment search tool. Journal of Molecular Biology, 215(3):403-410, 1990.

[44] M. Asayama, H. Suzuki, A. Sato, T. Aida, K. Tanaka, H. Takahashi, and M. Shirai. The rpoD1 gene product is a principal sigma factor of RNA polymerase in Microcystis aeruginosa K-81. Journal of Biochemistry, 120(4):752, 1996.

[45] Fabian Sievers, Andreas Wilm, David Dineen, Toby J Gibson, Kevin Karplus, Weizhong Li, Rodrigo Lopez, Hamish McWilliam, Michael Remmert, Johannes Söding, et al. Fast, scalable generation of high-quality protein multiple sequence alignments using Clustal Omega. Molecular Systems Biology, 7(1), 2011.

[46] C.G. Lerner and M. Inouye. Low copy number plasmids for regulated low-level expression of cloned genes in Escherichia coli with blue/white insert screening capability. Nucleic Acids Research, 18(15):4631, 1990.

[47] J.G. Scaife, J.S. Heilig, L. Rowen, and R. Calendar. Gene for the RNA polymerase sigma 
subunit mapped in Salmonella typhimurium and Escherichia coli by cloning and deletion. Proceedings of the National Academy of Sciences, 76(12):6510, 1979.

[48] K. Tanaka and H. Takahashi. Cloning and analysis of the gene (rpoDA) for the principal $\sigma$ factor of Pseudomonas aeruginosa. Biochimica et Biophysica Acta, 1089(1):113-119, 1991.

[49] D. Nilsson and E. Johansen. A conserved sequence in tRNA and rRNA promoters of Lactococcus lactis. Biochimica et Biophysica Acta (BBA), 1219(1):141-144, 1994.

[50] H. Tiberius Matern, J. Robert Klein, B. Henrich, and R. Plapp. Determination and comparison of Lactobacillus delbrueckii ssp. lactis DSM7290 promoter sequences. FEMS Microbiology Letters, 122(1-2):121-128, 1994.

[51] H.J. Boot, CP Kolen, F.J. Andreadaki, R.J. Leer, and P.H. Pouwels. The Lactobacillus acidophilus S-layer protein gene expression site comprises two consensus promoter sequences, one of which directs transcription of stable mRNA. Journal of Bacteriology, 178(18):5388, 1996.

[52] P.R. Jensen and K. Hammer. Artificial promoters for metabolic optimization. Biotechnology and Bioengineering, 58(2-3):191-195, 1998.

[53] A. McCracken, M.S. Turner, P. Giffard, L.M. Hafner, and P. Timms. Analysis of promoter sequences from Lactobacillus and Lactococcus and their activity in several Lactobacillus species. Archives of Microbiology, 173(5):383-389, 2000.

[54] I. Rud, P.R. Jensen, K. Naterstad, and L. Axelsson. A synthetic promoter library for constitutive gene expression in Lactobacillus plantarum. Microbiology, 152(4):1011, 2006.

[55] Y. Natori, Y. Kano, and F. Imamoto. Characterization and promoter selectivity of Lactobacillus acidophilus RNA polymerase. Biochimie, 70(12):1765-1774, 1988.

[56] R. Calendar, JW Erickson, C. Halling, and A. Nolte. Deletion and insertion mutations in the rpoH gene of Escherichia coli that produce functional sigma 32. Journal of Bacteriology, $170(8): 3479,1988$. 


\section{Figures}

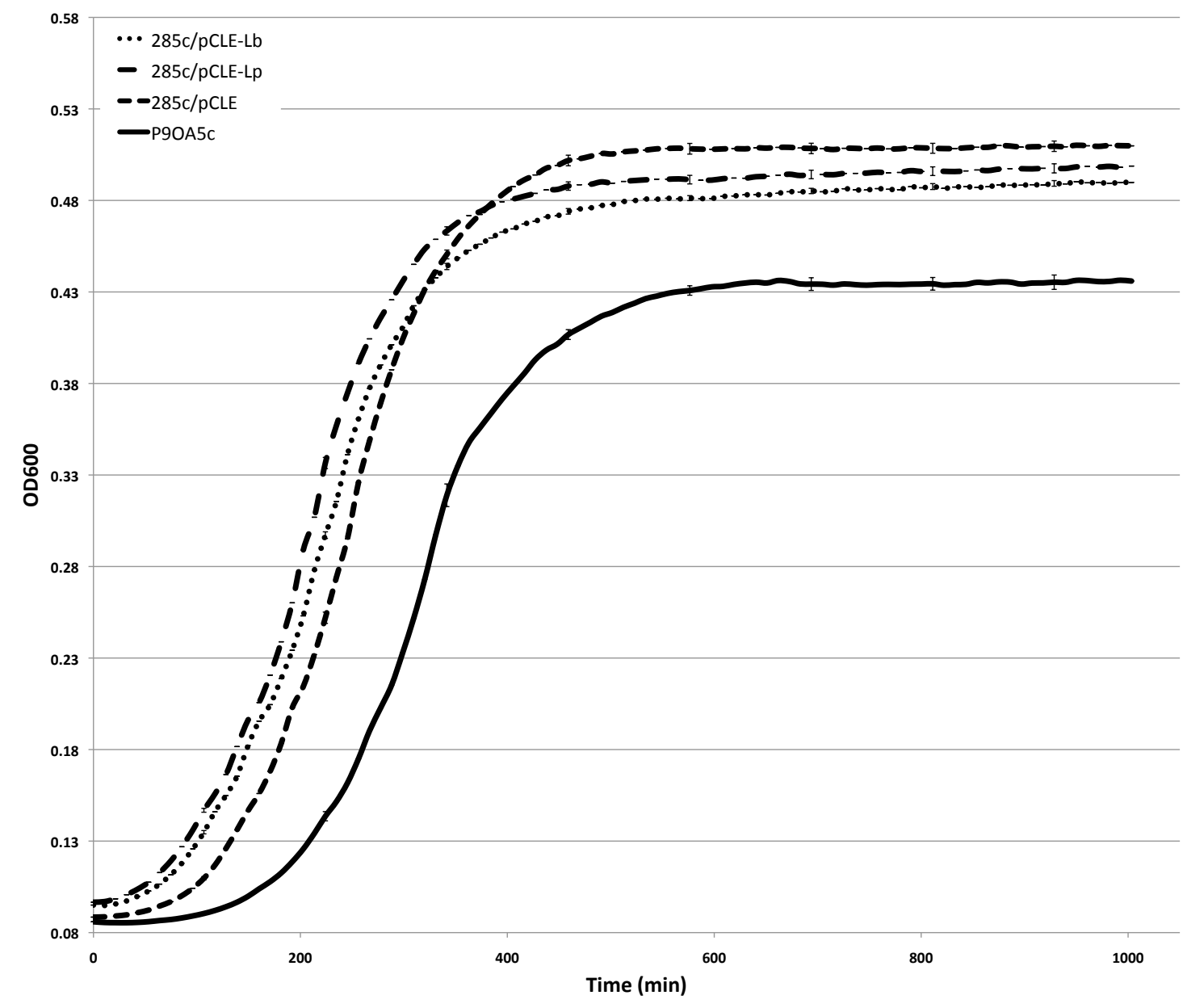

Figure 1: Growth of all 285c strains and the parent strain at $30^{\circ} \mathrm{C}$. Each strain was inoculated from overnights into LB with $25 \mu \mathrm{g} / \mathrm{ml}$ streptomycin (antibiotic omitted for P9OA5c) and cultured at $30{ }^{\circ} \mathrm{C}$ to determine the growth kinetics at the permissive temperature. As can be seen, the growth rates and the yields of the strains are similar. 


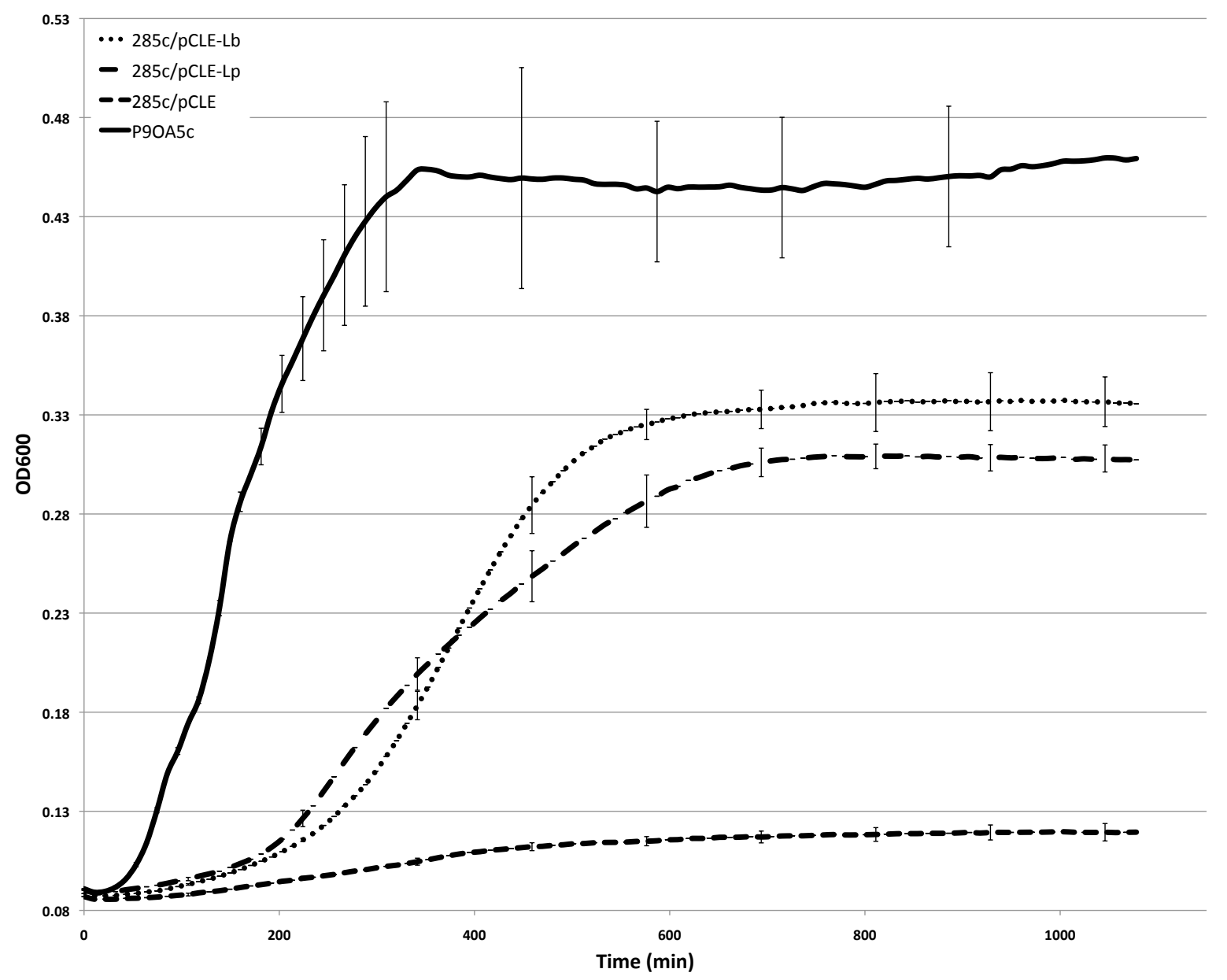

Figure 2: Complementation challenge at $42{ }^{\circ} \mathrm{C}$. Each strain was inoculated from overnights into LB with $25 \mu \mathrm{g} / \mathrm{ml}$ streptomycin (antibiotic omitted for P9OA5c) and cultured at $42{ }^{\circ} \mathrm{C}$ to determine if the heterologous sigma factors could complement a temperature sensitive defect in the native $\sigma^{70}$ protein. Robust growth is observed for 285c/pCLE-Lb and 285c/pCLE-Lp while the $285 \mathrm{c} / \mathrm{pCLE}$ strain is not viable at the elevated temperature. 
Ecoli

Ccrescentus
Bpertussis
Hpylori
Rprowazii
Maeruginosa
Bsubtilis
Iplantarum
Iplarum

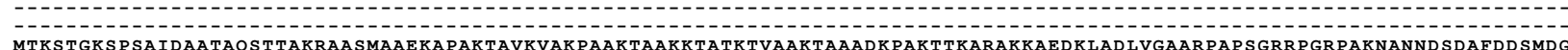

MTKS GKS PSAIDATAQSTTARRAASMAAKAPAKTAVVAKPAAKTAKKTATKVAAKTAAARPAKTTKAAKKAEDKLDIVGAARPASGRRRPGRAKNANNDDAFDDSMDEE

Ecoli
Ccrescentus
Bpertussis

-

Hpylori

G.
1

paeruginosa 1 i 1 - - MSNSN

Lplantarum
Lbrevis

- MARQTHET
$-M$ MARAKATT

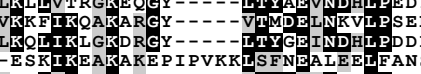

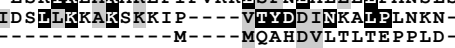

Ecoli

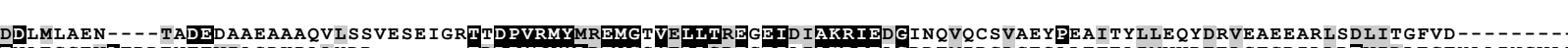

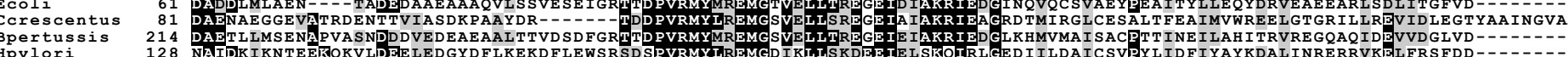

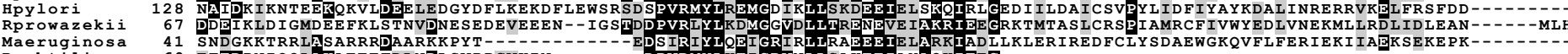

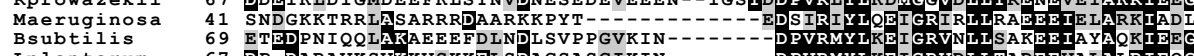

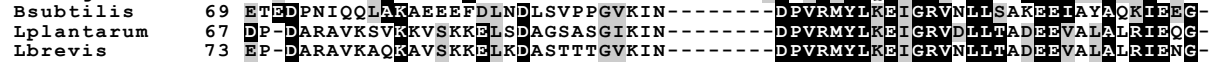

Ecoli

168 PNAEFDLAPTATHVGSELSQEDLDDDEDEDIEDDGDD------DSADDDNSIDPELAREKFAELRAQYVVTRDT IKAKGRSH-------ATAQEEILKLSEVFKQFRLVPRQFDYIVNSM

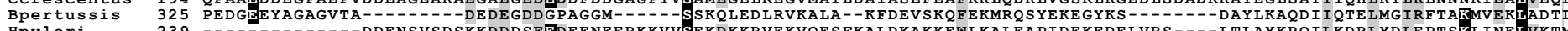

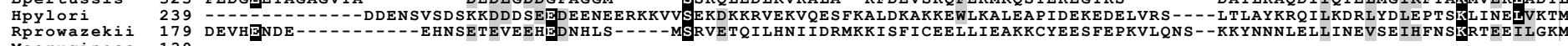

Msubtilis
Lplantarum
Litis

brevis

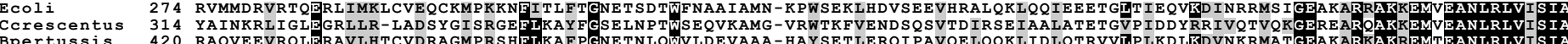

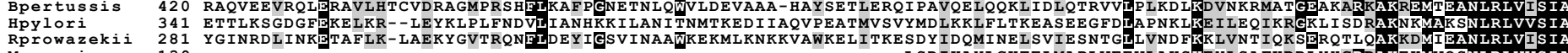

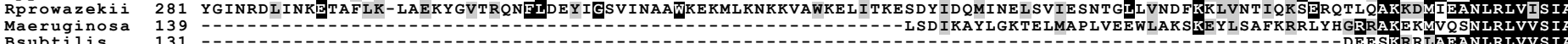

$\begin{array}{ll}\begin{array}{l}\text { Bubtilis } \\ \text { Lplantarum }\end{array} & 131 \\ \text { Lbrevis } & 128\end{array}$

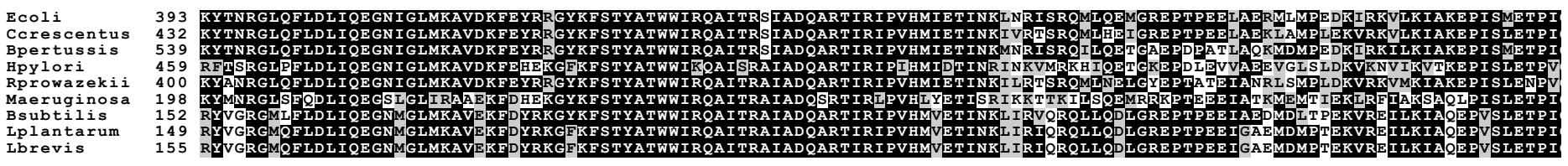

$5 c 01$

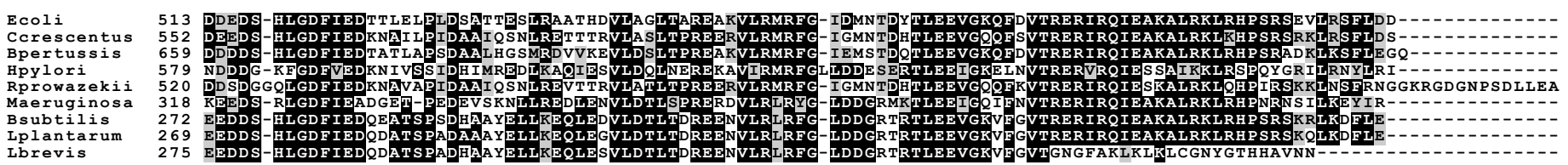

Figure 3: Alignments of E. coli $\sigma^{70}$ with heterologously expressed rpoD homologs from other organisms [27-31, 44]. Conserved residues are shaded in gray or black depending while Alignments were performed with Clustal Omega [45] and visualized using Boxshade. 